\title{
Comparison of Patients' Self-Diagnosis of Malaria and their Test Results from Medical Laboratory Analysis: The Case of Limbe Regional Hospital
}

\author{
Article by Achankeng Nkemasong Simon \\ Master of Public Health, Texila American University, Cameroon \\ Email: snkemasong@gmail.com
}

\begin{abstract}
Malaria remains one of the most wild spread infectious diseases of humankind, threatening approximately half the world's population and causing debilitating illness in more than 216 million people morbidity and mortality is particularly high in sub-Saharan Africa. Malaria is prevalent throughout Cameroon with transmission being affected by climate and geography, increased drug resistance and the lack of adequate vector control measles. It accounts for 40 to $45 \%$ of medical consultations, $57 \%$ of hospitalization days and $40 \%$ of mortality among children below 5 years. The objective of this study wee to determine the proportion of patients attending Limbe Regional Hospital whose self- diagnosis of malaria is confirm with Laboratory Analysis. A questionnaire was administered to the participants in a cross-section study. The respondent's consent was sought. Data was analyzed manually for chi square. The level of significant was set at $p>0.05$. in this study, $65.7 \%$ of the 105 participants successfully self diagnosed malaria. Also, $71.4 \%$ of the studied participants knew the true cause of malaria to be plasmodium parasite. The commonest signs and symptoms as mentioned by the respondent were as follows : 33(31.4\%) increased by body temperature , 23(21.9\%) headache ,12(11.429\%)body pains .12(11.4\%) sweating and 25(23.8\%) gave other symptoms .The level of education attained by respondents greatly influenced their ability to self-diagnosed malaria and to know the true cause of malaria $\left(x^{2}\right.$ calculated $=14.02, x^{2}$ Tabulated $=5.99, P<$ 0.05).Competent authorities need to intensify and implement proper health education messages on the risk factors of malaria in order to improve upon the understanding of disease in the nation.
\end{abstract}

\section{Introduction}

\section{Background and problem statement}

Malaria remains most wild spread infection disease of human kind, treating approximately half of the world's population and causing debilitating illness I more than 260 million people. Mobility and mortality is particularly high in sub Sahara Africa, with children below five years are greater risk Plasmodium falciparum, the deadliest of the malaria parasite species, kills a child every second in Africa and also cause significant havoc in highly endemic areas, substantially decreasing Gross domestic product (GDP) of affected countries relative to malarial free regions.

Malaria is prevented throughout Cameroon with transmission being affected by climate and geography increase drug resistance and lack of adequate vector control measures. In Cameroon, malarial account from 40 to $45 \%$ of medical consultation, $57 \%$ of hospitalization days and $40 \%$ of mortality amongst children below 5 years. In 1992, malarial accounted for $35 \%$ mortality in the general population in Cameroon. Currently, malarial dead rate per 100,000 population of age $0-4$ years was 89 and a hundred and three for all ages in Cameroon in 2008 .

Studies on factors associated with people's ability to self diagnose malaria, may provide additional understanding on early diagnosis of malaria are eventually lead to improve case management. Most of the studies review about people knowledge of malaria that some people still have misconception or about cases of malarial a study carry out in Hoima district in 
South American Journal of Public Health

Special Edition May 2016

Urganda reported that community members misconception about cause of malarial include, bedbugs, poor nutrition. A similar study which was carry out in Kampala city in Uganda also indicated $90 \%$ of the care givers know that mosquitoes cause malarial although they equally indicated other perceived causes such as drinking un-boiled water and respiratory illnesses. These misconception certainly have implications of community's malarial preventing behaviors and practices in a study among 1197 health service users in northern Cameroon only one percent identify mosquitoes as a source of transmission Einterz and Bates in 1997 also shows that most patience in Cameroon do not know when they have fever. Dickson and others in the North West region in Cameroon indicated that only $27.9 \%$ of their studied participate knew the true cause of malarial.

In the developing world, the vast majority of malarial cases in both adult and children are treated at home. Only if the case is very severe, that the health services are likely to be use for malarial treatment these may mean that the symptoms of the disease are know. However this is not always the case because Eintirz and Bate (1997) noted that a remarkable percentage $(87 \%)$ is patient age 15 years or over incorrect claim they had fever in Northern Region of Cameroon. Previous studies shows that general public's knowledge on the causes of materials and others determinates in Africa are poor.

The symptoms used by many people to diagnose and classify malaria are many and not necessarily the same as those used in biomedicine. In a study of Liberian children and mothers, it was found that for the study of population, malaria symptoms consisted of a constellation of bio-culturally defined signs. The expression used by the respondent: body cold all over, head hurting too bad, body hot all over, weak body all bones hurting, sweating a lot, hurting belle, belle sore to touch loss of appetite, throwing up, body jerks, been sick nauseated ,turning eyes dizziness , and other symptoms. Eleven malarial signs and symptoms where reported for children and 14 for mothers. In another studies in rural Ghana it was reported that Adangbe recognized malarial as a symptom complex locally know as asra. Asra is characterized by headache a rise in body temperature, chills, bitterness of the mouth, yellow eyes deeply colored urine, loss of appetite, body aches and pains, weakness and easy fatigability, vomiting, pallor of the palms and sole and cold sores around the moth.

Following the publication of Dickson and others in 2011 in a study carried out in Ndu area in the North West Region of Cameroon on "knowledge and practice relating to malaria: signs and symptoms cause and prevention "out of 253 participants, one hundred and fifty-six (6.7\%) participants know the symptoms of malaria to be fever and chills, $38(15.0 \%)$ to be headache, $35(13.8 \%)$ to be joint pains, $18(7.1 \%)$ to be vomiting and $6(2.4 \%)$ did not know any symptom of malaria. Malaria has symptoms that can, sometimes leads to misdiagnosis by many people. Even expert's needs to perform blood smear tests in order to confirm the presence of malaria parasites. Some personnel rely on clinical diagnosis and they use presumptive treatment. Across the world; however studies show that lay people generally identify malaria using similar symptoms

This study is aim at determining the proportion of patients who can successfully self diagnoses malaria using clinical sign and symptoms. Other purpose of this study is to determine the people knowledge on malaria as well to fine out factor that may influence people ability to self-diagnose malaria.

\subsection{Hypothesis}

More than $50 \%$ of patients that attend the Limbe Regional Hospital in can successfully self -diagnosing malaria using clinical signs and symptoms.

\subsection{Reseach objectives}

- Comparison of patients self diagnosis of malaria and their test result from medical laboratory analysis: the case of Limbe Regional Hospital 


\subsubsection{The Specific Objective}

- To asses people knowledge on the causes of malaria

- To determine the proportion of patients who successfully self-diagnose malaria using clinical signs and symptoms

- To determine the proportion of patients whose laboratory analysis result is positive for malaria after testing

- To compare the proportion of patients whose self-diagnosis of malaria and laboratory result match

\section{Literature review.}

\subsection{Introduction}

Despite efforts put in by the international community to reduce the burden of malaria in Africa, the disease especially that cause by plasmodium falciparum, is still a major health problem in sub- Saharan Africa and is responsible for over one million deaths annually. Mobility and mortality is particularly high in sub-Sahara Africa with children below five years at the greatest risk. In malaria -endemic countries, a large proportion of P. falciparum infection are asymptomatic, and asymptomatic carriers do not usually seek treatments for their infection and therefore, constitute a reservoir of parasite available for transmission by Anopheles mosquitoes. It is thought that long term asymptomatic carriage may represent a form of tolerance to the parasite in children, building up their immune response, thereby protecting the children from developing either a mild malaria attacks or a more severe one, by keeping their immunity effective.

In endemic areas, malaria accounts for over $25-35 \%$ of all outpatients visit, $20-45 \%$ of hospital admissions and $15-35 \%$ hospital death (240 the clinic spectrum of pediatric p. Falciparum infections range asymptomatic parasite carriage to a febrile disease that may develop into severe, life -threatening illnesses.

In Cameroon, malaria accounts for over $40 \%$ hospital attendance and therefore, takes an enormous tool on their lives in terms of medicine costs, day of labor lost and native effects on learning, especially by climate, geography increase drugs resistance and the lack of adequate vector control measures.

The life cycle of malaria parasite which occurs between the human and the female anopheles mosquito consists of three main cycles which includes the pre erythrocytic cycle and the erythrocytic cycle in human and the sporogonic cycle in the insect.

Clinical malaria can present with a range of symptoms such as fever, vomiting and convulsion, which are all associated with other infections. Typhoid fever has been shown to have similar sign and symptoms with malaria such as fever, headache, abdominal pains, relative bradycardis, splenomegaly and leucopenia. An association between malaria and typhoid fever was first describe in the medical literature in the middle of the $19^{\text {th }}$ century, and was name typhoid malaria fever by the united state army.

\subsection{The global situation of malaria}

Malaria constitute approximately $25 \%$ of the world's burden of disease, with an optimum effect of mortality and mobility in the tropical and sub-tropical region of the world, regardless of decades of earnest and conscientious activities on prevention and control. Effects of malaria could be long or short term and the global death attributed to the disease largely occur in over three billon of the worlds ' population that lives in malarious areas (33). The disease has been lined to consequences of broad rage some of which include: hearing impairment, visual impairment epilepsy, in addition to anemia, low birth weight and growth with retardation, especially in malaria associated with the plasmodium falciparum, the most affected being young children and pregnant women. Effects of malaria could be long or short term and the global death attributed to the diseases largely occurs in over three billon of the world's population that lives in malaria areas. The disease has been linked to consequences of 
broad range of which includes: hearing impairment, visual impairment and epilepsy, in addition to anemia, low birth rate and growth radiation, especially in malaria associated with Plasmodium falciparum. Malaria is a parasitic disease that is transmitted by and infected mosquitoes. The parasite also called plasmodium is a single cell organism, and transmission from one person to another. Is through the bite of an infected female anopheles mosquito, which require blood for development of its eggs. Despite many species of the Plasmodium genus, those responsible for the causes of human infection include $P$. falciparum $P$. vivax, $P$.ovale, and $P$. malariae with $P$. falciparum accounting for most death.

\subsubsection{Malaria situation in Cameroon}

Malaria situation in Cameroon is not different from that of other endemic sub- Saharan African counties. Cameroon is a country with vast diversity in vegetation and topological land forms where natural environment covers three ecological and epidemiological zones i.e. equatorial region in which malaria is endemic with transmission throughout the year, guinea savanna or tropical region in which transmission is seasonal between three to six months a year and Sahel savanna where the transmission season and duration is less than three months ) all of which is $95 \%$ endemic of malaria the entire country is endemic to malaria but different regions are exposed to varied levels of transmission (13.5million and 5.5 individuals are exposed to high and low transmission respectively). About 8.3 million people live in royal areas which form $43 \%$ of total population. Children less than 5 years represent $16 \%(3.117$ million) of Cameroon's total population approximately 19 million and there are the most threaten by malaria. Several anopheleses have been noted and identify as host of mainly falciparum and vivax species that transmit the disease.

\subsection{The life circle of malaria parasite}

The malaria parasite has a complex, multistage life cycle occurring within two living beings, the vector mosquitoes and the vertebrate host. The survival and development of the parasite within the invertebrate and vertebrate host, intercellular and extra cellular environment, is made possible by a tool kits of more than five thousand and their specialized proteins that helps the parasite to invade and row with in the multiple cell types and to invade host immune responses. The parasite passes through several stages of development such as the sporozoites (the infectious form injected by the mosquitoes), the merozoites ( the stage invading erythrocytes), an trophozoites (the form multiplying in erythrocytes) gametocytes ( sexual stages ) and all these stages have their own unique sharps and structures and protein complements . The surface proteins and metabolic part ways keep changing during this stages during these stages that help the parasite to invade the immune clearance, while also creating problems for the development of drugs and vaccines.

\subsubsection{The sporogony circle with in the mosquitoes}

Mosquitoes are the definitive host for malaria parasite; where the sexual phrase of parasite life circle occurs. The sexual phrase is call sporogony and result in the development of innumerable infecting forms of the parasite within the mosquitoes that induce disease in the human host following their injection with the mosquitoes bite.

When the female anopheles draws a blood meal from an individual infected with malaria, the male and female gametocytes of the parasite fine their way into the gut of the mosquitoes. The molecular and circular changes is the gametocytes help the parasite to quickly adjust to the insect host from the warm blooded human host and then to initiate the sporogonic cycle. The male and female gametes fuse in the mosquito's guts to form zygotes, which subsequently develop into actively moving ookinetes that burrow into the mosquito midgut wall to develop into oocysts. Growth and division of each oocysts produces thousands of active haploid forms called sporozoites. After the sporogonic phase of 8-15 days, the oocyst bursts and releases sporozoites into the body cavity of the mosquito, from where they travel to and invade the mosquito salivary glands. When the mosquito thus 
loaded with sporozoites takes another blood meal, the sporozoites get injected from its salivary glands into the human bloodstream, causing malaria infection in the human host. It has been found that the infected mosquito and the parasite mutually benefit each other and thereby promote transmission of the infection. The Plasmodium infected mosquitoes have a better survival and show an increased rate of blood-feeding, particularly from an infected host.

\subsubsection{Schizogony in human host:}

Man is the intermediate host for malaria; where in the asexual phase of the life cycle occurs. The sporozoites inoculated by the infested mosquito initiate this phase of the cycle from the liver, and the latter part continues within the red blood cells, which results in the various clinical manifestations of the lymphatic, and some others find a blood vessel. The sporozoites disease.

\section{3.3 Pre-Erythrqcytic phase - schizogony in the liver:}

With the mosquito bite, tens to a few hundred invasive sporozoites are introduced into the skin. Following the intra-dermal deposition, some sporozoites are destroyed by the local macrophages, some enter the that enter a lymphatic vessel reach the draining lymph node where in some of the sporozoites partially develop into exoerythrocytic stages and may also prime the $\mathrm{T}$ cells to mount a protective immune response. The sporozoites that find a blood vessel reach the liver within a few hours. It has recently been shown that the sporozoites travel by a continuous sequence of stick-and-slip motility, using the thrombospondin-related anonymous protein (TRAP) family and an actin-myosin motor, The sporozoites then negotiate through the liver sinusoids, and migrate into a few hepatocytes, and then multiply and grow within parasitophorous vacuoles. Each sporozoite develops into a schizont containing 10,000-30,000 merozoites (or more in case of P. falciparum). The growth and development of the parasite in the liver cells is facilitated by a favorable environment created by the circum sporozoite protein of the parasite. The entire pre-eryhrocytic phase lasts about 5-16 days depending on the parasite species: on an average 5-6 days for $P$. falciparum, 8 days for P. vivax, 9 days for $\mathrm{P}$. ovale, 13 days for $\mathrm{P}$, malariae. The preerythrocytic phase remains a "silent" phase, with little pathology and no symptoms, as only a few hepatocytes are affected. This phase is also a single cycle, unlike the next, erythrocytic stage, which occurs repeatedly. The merozoites that develop within the hepatocyte are contained inside host cell-derived vesicles called merosomes that exit the liver intact, thereby protecting the merozoites from phagocytosis by Kupffer cells. These merozoites are eventually released into the blood stream at the lung capillaries and initiate the blood stage of infection there on. In P. vivax and P. ovale malaria, some of the sporozoites may remain dormant for months within the liver. Termed as hypnozoites, these forms develop into schizonts after some latent period, usually of a few weeks to months. It has been suggested mat these late developing hypnozoites are genotypically different from the sporozoites that cause acute infection soon after the inoculation by a mosquito bite, and in many patients causes relapses of the clinical infection after weeks to months.

\subsubsection{Erythrocytic schizogony - centre stage in red cells.}

Red blood cells are the 'centre stage ${ }^{1}$ for the asexual development of the malaria parasite. Within the red cells, repeated cycles of parasitic development occur with precise periodicity, and at the end of each cycle, hundreds of fresh daughter parasites are released that invade more number of red cells. The merozoites released from the liver recognize, attach, and enter the red blood cells (RBCs) by multiple receptor-ligand interactions in as little as 60 seconds. This quick disappearance from the circulation into the red cells minimizes the exposure of the antigens on the surface of the parasite; thereby protecting these parasite forms from the host immune response, the invasion of the merozoites into the red cells is facilitated by molecular interactions between distinct ligands on the merozoites and 
South American Journal of Public Health

Special Edition May 2016

host receptors on the erythrocyte membrane. P, vivax invades only Duffy blood grouppositive red cells, using the Duffy-binding protein and the reticulocyte homology protein, found mostly on me reticulocyles. The more virulent $P$. falciparum uses several different receptor families and alternate invasion pathways that are highly redundant. Varieties of Duffy binding-like (DBL) homologous proteins and the reticulocyte binding-like homologous proteins of P. falciparum recognize different $\mathrm{RBC}$ receptors other than the Duffy blood group or the reticulocyte receptors- Such redundancy is helped by the fact that P- falciparum has four Duffy binding-like erythrocyte-binding protein genes, in comparison to only one gene in the DBL-EBP family as in the case of P. vivax, allowing $P$. falciparum to invade any red cell. The process of attachment, invasion, and establishment of the merozoite into the red cell is made possible by the specialized apical secretary organelles of the merozoite, called the micronemes, rhoptries, and dense granules. The initial interaction between the parasite and the red cell stimulates a rapid "wave" of deformation across the red cell membrane, leading to the formation of a stable parasitehost cell junction. Following this, the parasite pushes its way through the erythrocyte bilayer with the help of the actin-myosin motor, proteins of the thrombospondin-related anonymous protein family and aldolase, and creates a parasitophorous vacuole to seal itself from the host-cell cytoplasm, thus creating a hospitable environment for its development within the red cell. At this stage, the parasite appears as an intracellular "ring". Within the red cells, the parasite numbers expand rapidly with a sustained cycling of the parasite population. Even though the red cells provide some immunological advantage to the growing parasite, the lack of standard biosynthetic pathways and intracellular organelles in the red cells tend to create obstacles for the fast-growing intracellular parasites. These impediments are overcome by the growing ring stages by several mechanisms: by restriction of the nutrient to the abundant hemoglobin, by dramatic expansion of the surface area through the formation of a tuba vesicular network, and by export of a range of remodeling and virulence factors into the red cell. Hemoglobin from the red cell, the principal nutrient for the growing parasite, is ingested into a food vacuole and degraded. The ammo acids thus made available are utilized for protein biosynthesis and the remaining toxic heme is detoxified by heme polymerase and sequestrated as hemozoin (malaria pigment). The parasite depends on anaerobic glycolysis for energy, utilizing enzymes such as pLDH, Plasmodium aldolase etc. As the parasite grows and multiplies within the red cell, the membrane permeability and cytosolic composition of the host cell is modified. These new permeation pathways induced by the parasite in the host cell membrane help not only in the uptake of solutes from the extracellular medium but also in the disposal of metabolic wastes, and in the origin and maintenance of electrochemical ion gradients. At the same time, the premature hemolysis of the highly permeabilized infected red cell is prevented by the excessive ingestion, digestion, and detoxification of the host cell hemoglobin and its discharge out of the infected RBCs through the new permeation pathways, thereby preserving the osmotic stability of the infected red cells.

The erythrocytic cycle occurs every 24 hours in case of P. knowtesi, $48 \mathrm{~h}$ in cases of P. falciparum, P. vivax and P. ovale and $72 \mathrm{~h}$ in case of P. malariae.

During each cycle, each merozoite grows and divides within the vacuole into 8-32 (average 10) fresh merozoites, through the stages of ring, trophozoites and schizont. At the end of the cycle, the infected red cells rupture, releasing the new merozoites that in turn infect more RBCs, with sun bridled growth, the parasite numbers can rise rapidly to levels as high as 1013 per host.

A small proportion of asexual parasites do not undergo schizogony but differentiate into the sexual stage gametocytes. These male or female gametocytes are extracellular and nonpathogenic and help in transmission of the infection to others through the female anopheles mosquitoes, where in they continue the sexual phase of the parasite's life cycle. Gametocytes of P. vivax develop soon after the release of merozoites from the liver, whereas 
in case of P. falciparum $t$ the gametocytes develop much later with peak densities of the sexual stages typically occurring 1 week after peak asexual stage densities.

\section{4: Pathogentcitv of malaria}

It has been observed that most severe effects of malarial infection in stable areas are seen in children aged 6 months to 5 years. The pathological changes in malaria are related to the development of asexual parasites in human's blood. The release of malaria antigens, pigment and toxins at the time of schizonts rupture give rise to many pathological events. Pathology of malaria manifests in individual organs of the system in different ways. In the early stages of infection, malaria fever is irregular or continues. As schizogony cycles synchronized, fever begins to recur at regular intervals. As malaria illness continues, the following pathogenicity may be notice in infected individuals.

- AINAEMIA. This can be severe and occur rapidly particularly in young children. In the case of Falciparum, this is due to destruction of parasitized red cells. They are also a reduction in the production of red cells in the bone marrow. An immune destruction of non-parasitized red cells also occurs.

- HYPOGLYCAEMIA. Decrease blood glucose level. This is common in children and pregnant women with severe falciparum malaria and as a complication of those treated with quinine and quinidine (drugs thought to stimulate the pancreas to produce insulin which lowers blood glucose levels.

- MALARIA HAEMOGLOBINUREA (Formerly known as black water fever). That is hemoglobin in urea as a result of malaria infection. This occurs in severe malaria and in association with glucose 6 phosphate dehydrogenase deficiency (G6PD). Here, they are rapid and massive intravascular hemolysis of both parasitized and non-parasitized red cells resulting to hemoglobin being passed out in urine (hemoglobinurea) and a total fall in hemoglobin. The urine appear dark red to brown black due to the presence of free hemoglobin and also certain proteins, hyaline and granular casts, and epithelial debris.

- CEREBRAL MALARIA, This is a common cause of coma and death in falciparum malaria and particularly in children and non-immune adult. In this case, many parasitized cells are found in the capillaries of the brain and other organs and in late stages hemorrhaging from small blood vessels can occur therefore preventing regular blood flow and can cause stroke in the case of brain.

- HYPER REACTIVE MALARIA SPLENOMEGALY. In this situation, they are defective regulation of immune response associated with recurrent infection by P. Falciparum, P. Vivax, or P, Malariae. Those affected are immune adults in malaria endemic areas. Here, they are high level of IgM malaria antibody, and circulating immune complexes, and a moderately enlarged liver. Here, the white and platelet cell counts for the patient are very low and malaria parasites are rarely seen in blood films.

- Other complications of malaria infection may include: Abortion, still birth, premature labor or low birth weight, pulmonary oedema. These occur on pregnant women living in areas of unstable malaria. In HIV infected individuals, they is more frequent and severe malaria, anemia, placenta malaria, low birth weight and poor infant survival. Malaria is thought to increase HIV replication and increase risks of mother to child transmission of HIV, Nephrotic syndrome which may progress to renal failure can also occur in the case of P. malariae infection.

\subsection{Co infection of malaria and typhoid fever}

Malaria is caused by obligate intracellular parasites, which live in host crythrocytes and remodel these cells to provide optimally for their own needs. Typhoid fever is a systemic infectious disease characterized by an acute illness, with the first typical 
South American Journal of Public Health

Special Edition May 2016

manifestations of which being fever, headache, abdominal pain, relative bradycardia, splenomegaly, and leucopenia. An association between malaria and typhoid fever was first described in the medical literature in the middle of the 19th century, and was named typhomalarial fever by the United States Army (Smith, 1982a). In the last 20 years, this relationship between malaria and salmonellae has been confirmed by additional studies from Africa that largely describe a higher incidence of non-typhoid salmonella bactcracmia among patients with malarial parasitaemia. Clinicians have had difficulty in differentiating typhoid fever from malaria because of some overlapping clinical features. Because the inability of physicians to clinically differentiate these two entities, they used the term 'typhomalariar' as a diagnosis for acute fevers without localizing signs. Osier (1892) clearly differentiated malaria from typhoid fever by clinical criteria alone. By recognizing and appreciating the characteristic clinical features, we were able to differentiate malaria from typhoid fever. His observations remain valid and useful today, Osier also cited Malaria begins with multiple shaking chills, whereas typhoid fever begins with a single morning shaking chill In malaria, chills are followed by spiking fevers. Except for the initial shaking chill, chills are not common with typhoid fever. In malaria, chills precede produces fever followed by profuse diaphoresis and profound malaise followed by complete recovery between attacks.

\subsection{Malaria diagnosis}

\subsubsection{Clinical criteria for diagnosis}

Clinically, malaria is characterized by fever as a result of the released of toxins which stimulates the released of cytokines from white blood cells and other cells of the body. The first symptoms of malaria (most often fever, chills, sweats, headaches, muscle pains, nausea and vomiting) are often not specific and are also found in other diseases (such as influenza and other common viral infections). Likewise, the physical findings are often not specific (elevated temperature, perspiration, tiredness). In severe malaria \{typically caused by P. falciparum), clinical findings (confusion, coma, neurologic focal signs, severe anemia, respiratory difficulties) are more sinking and may increase the suspicion index for malaria. P, falciparum infections are potentially life-threatening because the proportion of red blood cells containing parasites can be greater than $10 \%$ which results in venous thrombosis or sludging in the capillaries. Complications of inadequately treated falciparum malaria include anemia, renal failure, shock, adult respiratory distress syndrome, encephalopathy, and acidosis. Disease caused by the other malarial species is rarely fatal, and can be quite mild. In Southeast Asia where P. knowlesi infection more commonly occurs, it may be misdiagnosed as less severe $\mathrm{P}$, malariae by its appearance but, is more clinically similar to falciparum malaria in terms of severity.

\subsection{2, Laboratory criteria for diagnosis}

Malaria must be recognized promptly in order to treat the patient in time and to prevent further spread of infection in the community. Though malaria can be suspected based on the patient's symptoms and the physical findings at examination, for definitive diagnosis to be made, laboratory tests must demonstrate the malaria parasites or their components. In the laboratory, malaria can be detected by the following methods:

- Detection of malaria parasites in thick or thin peripheral blood films

- Detection of circulating malaria-specific antigens using rapid diagnostic test (RDT).

- Detection of species specific parasite DNA in a sample of peripheral blood using a polymerase chain reaction test (PCR).

- Other diagnosis may include: The measurement of hemoglobin or packed cell volume (PCV) which may suggest malaria with heavy parasitaemia, Measurement of blood glucose, total white cell count and platelets count may indicate severe faIciparum. Coagulation test for abnormal bleeding for suspicion of falciparum, 
free hemoglobin test in urine if black water fever is suspected, testing urine for protein when Nephrotic syndrome is suspected and screening for $G_{6} P D$ deficiency before treating a patient with an oxidant drug such as premaquine.

\subsection{Control and prevention of malaria}

In order to reduce malaria death cases, competent strategies need to be put in place in order to help the expose individuals to control and possibly prevent themselves from the disease. Competent authorities can equally help in controlling and prevention of malaria in various areas through the following:

- Providing accessible malaria diagnostic facilities and affordable, accessible drugs to treat promptly active infections and prevent malaria in those at risks such as pregnant women and non-immune persons visiting endemic areas. Also increase public awareness of the dangers of malaria and how to reduce contact with the mosquitoes.

- Avoiding mosquitoes' bites by using long lasting insecticides treated bed nets, screening windows and doors with mosquitoes netting, wearing protective clothing and using mosquitoes' repellants,

- Preventing the breeding of mosquitoes by removing surface water, filling in ponds, drainage ditches, and potholes, altering breathing sites, spraying breathing sites and destroying adults' mosquitoes using effective chemicals.

- Acting more rapidly and effectively to control epidemics in complex emergency situations.

The Cameroon government has equally put in place some strategies in fighting against malaria, in order to effectively control and eradicate malaria from Cameroon. The ministry of Public Health, with the assistance of WHO, and others malaria stakeholders such as, Roll Black Malaria, Fobang Foundation, IRESCO, MIM and many others, have been working hand in hand on implementing malaria preventive methods in Cameroon. On $20^{\text {th }}$ August 2011, the Prime Minister, Philemon Yang, launched the campaign to distribute freely over 8,654,731 Long Lasting Insecticide-Treated bed nets in the 10 regions of Cameroon. The government has made available free malaria drugs for children aged between zeros to five years, as well as the prophylactic drugs for pregnant women. The government has also trained peer, educators who move from door to door and give education concerning the disease to people.

\section{Material and methods}

\subsection{Study area}

This study was carried out in the Limbe Regional Hospital. The hospital is situated at Mile 1 in Limbe, Southwest Region Cameroon. Limbe is the regional capital of the South West Region. It is a cosmopolitan city with a heterogeneous population of about 1,237,500 composed of people of different socio-economic levels and ethnic groups from all over Cameroon and other nationalities. Limbe features a tropical wet and dry climate with constant temperatures throughout the year. Temperatures are not quite as hot as one would expect for a city located near the equator. Limbe features a lengthy wet season, covering a six month span between May and October. However, there is a noticeable increase in precipitation within the wet season, seen during the months of July and August.

\subsection{Study population}

Participants consisted of in and out patients who attended this health unit and were suspected of having malaria by medical doctors, and the asked to go to the laboratory for diagnosis.

\subsection{Recruitment criteria}

Participants were strictly those who had consulted in this health unit. Participants were able 
South American Journal of Public Health

Special Edition May 2016

to fill or answer the questions on the structures questionnaire. Each participant was interviewed using either a structured questionnaire or given the questionnaire to be filled.

\subsection{Methods}

\subsubsection{Materials.}

The following materials were used in the study; gloves, cotton wool, alcohol, methanol, lancets, slides, giemsa stain, staining trough, staining rack, microscope, immersion oil, slide spreader, slide box, thermometer, a questionnaire and a note book.

After drying, the thick films were examined for malaria parasites microscopically using $10 \mathrm{x}$ eyepieces and $100 \mathrm{x}$ immersion objectives. The thick films were used for quantification of parasites as follows as stated by WHO as shown.

\subsubsection{Examination for malaria parasites}

The thick and thin blood films were made from fingertip blood collected from the participants on the same slide and then allowed to air dry. The slides were placed on a staining trough and the thin films were fixed with methanol for 2 minutes. The thick and thick films were stained using 10 percent diluted solution of giemsa for 10 minutes. The slides were then washed in running tap water and left to air dry

After drying, the thick films were examined for malaria parasites microscopically using 10x eyepieces and 100x immersion objective. The thick films were used for quantification of parasites as follows as stated by $\mathrm{WHO}$ as shown.

$$
\text { Parasite per microliter }(\mu l)=\frac{\text { WBC count x parasites counted against 100WBC }}{100}
$$

This is as stipulated by WHO. A hundred high power microscopic fields were examined for each slide before considering the slide been negative.

\subsubsection{Body temperature}

The auxiliary body temperature of the participants was recorded using a clinical thermometer. Participants with temperature above 37 degrees were being considered as febrile.

\subsection{Sample Size}

Sample size calculation

The prevalence of various malaria complications extremely differs with the setting, seasons and is very dependent of the genetic variations of the population involved (Labie and Tamouza, 2004). The sample size for this study was calculated using the following formula (D'Agostino et al, 2006).

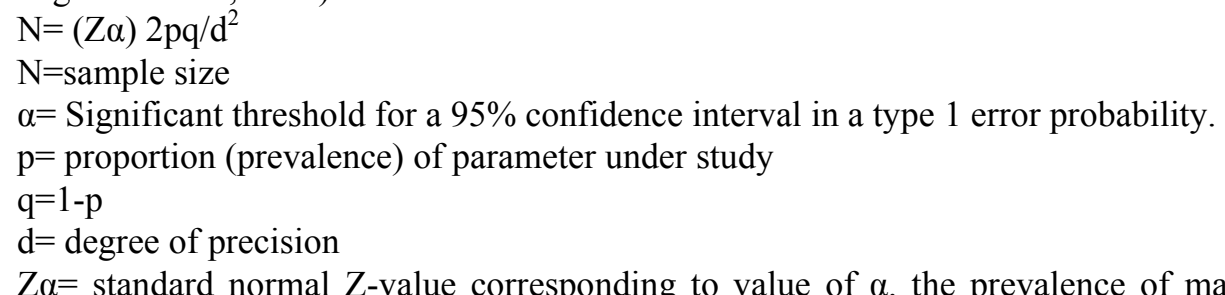

$\mathrm{Z} \alpha=$ standard normal $Z$-value corresponding to value of $\alpha$. the prevalence of malaria in Cameroon from WHO records 2011 is that, in this calculation we 1,845,691 Cameroonians are infected with malaria parasite each year, and the estimated population of Cameroon is $19,598,889$ giving a prevalence of approximately $9 \%$ and hence, gives the maximum sample size required for any given precision.
$\mathrm{a}=0.05$
$\mathrm{Z} \alpha=1.96$
$q=91 \%$
$\mathrm{p}=9 \%$
$\mathrm{d}=5 \%$ 


$$
\text { Sample size }-\frac{(1.96) 2(0.09)(0.91)}{(0.05)^{2}}-126 \text { participants }
$$

\subsection{Analysis of results:}

The results obtained from this study were analyzed manually for proportions. The level of significance was designated at $\mathrm{p}<0.05$. Charts and tables were also used for illustration of some of the results.

\section{Results}

A total of 105 participants participated in this study. Participants consisted of 50 males (47.6\%) and 55 females $(52.3 \%)$. The body temperature of participants ranged from $36^{\circ} \mathrm{C}$ to $39^{\circ} \mathrm{C}$. The ages of the participants ranged from $14-60$ years. The educational status of the participants varied from primary $20(19.0 \%)$, secondary $54(51.4 \%)$ and university, 25 (23.7\%). The occupations of the participants also varied from students 27 (25.7\%), civil servants $20(19.0 \%)$ and others $46(43.8 \%)$. Out of the 105 participants who took part in this study, 69 successful self-diagnosed malaria using clinical signs and symptoms, given a percentage of $65.7 \%$. Different signs and symptoms of malaria were sighted by the participants. Most of the participants $75(71.4 \%)$ knew the correct cause of malaria to be plasmodium parasite.

\subsection{Malaria self-diagnosis by participants and occupation}

Out of the studied participants who successfully self-diagnosed malaria, 27 (39.1\%) were students, $16(23.2 \%)$ were civil servants and $26(37.7 \%)$ had other occupations. They were no statistical significance difference in the different rate at which participants could selfdiagnosed malaria based on occupations as $p>0.05$ as shown in table one below. This means that participants had equal chances of self-diagnosing malaria with respect to their various occupations.

Table 1 ability of participants to self-diagnose malaria based on occupations.

\begin{tabular}{|c|c|c|c|c|}
\hline Occupation & Students & Civil servants & Others & $\mathrm{N}^{\mathrm{o}}$ tested \\
\hline Positive self diagnosis & $27(39.1 \%)$ & $16(23.2 \%)$ & $26(37.7 \%)$ & $69(65.7 \%)$ \\
\hline Negative self diagnosis & $12(33.3 \%)$ & $4(11.1 \%)$ & $20(55.6 \%)$ & $36(34.3 \%)$ \\
\hline $\mathrm{N}^{\mathrm{o}}$ tested & $39(37.3 \%)$ & $20(19.0 \%)$ & $46(43.8 \%)$ & $105(100 \%)$ \\
\hline
\end{tabular}

$\left(x^{2}\right.$ calculated $=3.71, x^{2}$ tabulated $=5.991$, degree of freedom $=2$, at $\left.\mathrm{p}=0.16\right)$

\subsection{Malaria self-diagnoses by participants based on their educational status}

Of the total number of participants who successfully self-diagnosed malaria, $6(8.7 \%)$ had attained primary level of education, $38(55.0 \%)$ had attained secondary level of education, 25 (36.2\%) had attained university level of education. It was evident from this study that education greatly influences people's ability to self-diagnosed malaria as $p<0.05$ with those in secondary level having the highest proportion as shown in table two below.

Table 2: Ability of participants to self-diagnosed malaria based on educational status.

\begin{tabular}{|c|c|c|c|c|}
\hline Educational status & Primary & Secondary & University & $\mathrm{N}^{\mathrm{o}}$ tested \\
\hline Positive self-diagnosis & $6(8.7 \%)$ & $38(55.1 \%)$ & $25(36.2 \%)$ & $69(65.7 \%)$ \\
\hline Negative self-diagnosis & $14(38.9 \%)$ & $16(44.4 \%)$ & $6(16.7 \%)$ & $36(34.4 \%)$ \\
\hline $\mathrm{N}^{\mathrm{o}}$ tested & $20(19.0 \%)$ & $24(22.9)$ & $31(29.5 \%)$ & $105(100 \%)$ \\
\hline
\end{tabular}


South American Journal of Public Health

Special Edition May 2016

\subsection{Malaria self-diagnosis by participants based on respective ages.}

The ranges of participant's ages who gave a successful self-diagnosis of malaria were as follows: $11(15.942 \%)$ were aged less than $21,48(69.565 \%)$ were aged between 21 to 45 , while $10(14.493 \%)$ were aged greater than 45 . Though those within the age range of 21-45 had the highest frequency in successful self-diagnosed malaria, they was no statistical significance difference between the different rates at which participants could self-diagnosis malaria based on age with $\mathrm{p}>0.05$ as shown in table three below.

Table 3: Participant's self-diagnosis of malaria based on age

\begin{tabular}{|c|c|c|c|c|}
\hline Age & $<21$ & $21-45$ & $>45$ & $\mathrm{~N}^{\mathrm{o}}$ tested \\
\hline Positive self-diagnosis & $11(16.0 \%)$ & $48(69.6 \%)$ & $10(14.5 \%)$ & $69(65.7 \%)$ \\
\hline Negative self-diagnosis & $9(25 \%)$ & $25(69.4 \%)$ & $2(55.6 \%)$ & $36(34.3 \%)$ \\
\hline $\mathrm{N}^{\mathrm{o}}$ tested & $20(19.0 \%)$ & $75(71.4 \%)$ & $12(11.4 \%)$ & $105(100 \%)$ \\
\hline
\end{tabular}

$\left(x^{2}\right.$ calculated $=2.44, x^{2}$ tabulated $=5.199$, degree of freedom $=2$, at $\left.\mathrm{p}=0.30\right)$

\subsection{Malaria self-diagnoses based on respective sexes of the participants}

Based on this study, among those who successfully self-diagnosed malaria, 36 (52.174\%) were males and $33(47.826 \%)$ were females. It is evident from this study that they was no statistical significance difference between age and self-diagnosing malaria between various participants with $\mathrm{p}>0.05$ as shown in table four below, though males had the highest frequency compare to females.

Table 4: Participant's self-diagnosis of malaria based on sex

\begin{tabular}{|c|c|c|c|}
\hline Sex & Male & Female & $\mathrm{N}^{\circ}$ tested \\
\hline Positive self-diagnosis & $36(52.2 \%)$ & $33(47.8 \%)$ & $69(65.7 \%)$ \\
\hline Negative self-diagnosis & $14(38.9 \%)$ & $22(61.1 \%)$ & $36(34.3 \%)$ \\
\hline $\mathrm{N}^{\mathrm{o}}$ tested & $50(47.6 \%)$ & $55(57.8)$ & $105(100 \%)$ \\
\hline
\end{tabular}

$\left(x^{2}\right.$ calculated $=1.46, x^{2}$ tabulated $=3.841$, degree of freedom $=1$, at $\mathrm{p}=0.23$

\subsection{Participants knowledge on the true caused of malaria based on their educational status.}

Of the studied participants who knew the true cause of malaria, 33(44.0\%) had attained university level of education, 38(50.7\%) had attained secondary level of education, 4(5.3\%) had attained primary level education. The level of education greatly influenced participants ability to know the true cause of malaria as they was a statistical significance difference with $\mathrm{p}<0.05$ as shown in table five below.

Table 5: Participant's knowledge on the signs and symptoms of malaria based on educational status.

\begin{tabular}{|c|c|c|c|c|}
\hline Educational status & Primary & Secondary & University & $\mathrm{N}^{0}$ tested \\
\hline True caused of malaria & $4(5.3 \%)$ & $38(50.7 \%)$ & $33(44 \%)$ & $75(71.4 \%)$ \\
\hline False caused of malaria & $14(46.7 \%)$ & $14(46.7 \%)$ & $2(6.7 \%)$ & $30(28.6 \%)$ \\
\hline $\mathrm{N}^{\circ}$ tested & $18(17.1 \%)$ & $52(49.5)$ & $35(33.3 \%)$ & $105(100 \%)$ \\
\hline
\end{tabular}

$\left(x^{2}\right.$ calculated $=15.75, x^{2}$ tabulated $=5.199$, degree of freedom $=2$, at $\left.\mathrm{p}=0.00\right)$

Table 6: the frequency of signs and symptoms of malaria as stated by the studied participants.

\begin{tabular}{|c|c|}
\hline Signs and symptoms of malaria & Number (\%) \\
\hline Increase body temperature & $33(31.4)$ \\
\hline Others & $25(24.0)$ \\
\hline Headache & $23(21.9)$ \\
\hline Body pains & $12(11.4)$ \\
\hline Sweating & $12(11.4)$ \\
\hline
\end{tabular}




\section{Discussion, conclusion and recommendations}

\subsubsection{Discussion}

This study on the ability of people to self-diagnosed malaria based on clinical signs and symptoms is of great public health importance because it investigates the various signs and symptoms that people use in the diagnosis of malaria, which may sometime differ with those use by medical Practisionals. It also investigates the knowledge of the participants on cause of the disease. We found out in this study that, of the 105 participants who participated in this study, 69 participants successfully self-diagnosed malaria based on their clinical signs and symptoms, giving a percentage of $65.7 \%$. most of the clinical signs and symptoms that different participants used in the diagnosis of the disease ties with WHO own diagnosis which states that a patient with malaria has increase body temperature, and also with the presence of malaria parasite in the blood film which leads to the expression of certain clinical signs and symptoms, this is also similar to the diagnostic criteria that CDC stat for diagnosing malaria.

In Table1, we see the frequency of participants to self-diagnosed malaria based on occupations. Though statically not significant, those whose occupations were student had the highest proportion of self-diagnosing malaria in this study compared to those whose occupations were civil servants. This could be due to the fact that they had the highest proportion of the studied participants. Also, those who sighted other occupations such as business, house wife and guards had the least diagnosis compare to those who whose occupations were students and this could be due to the knowledge they have learn in school about the disease. Those whose occupation were civil servant diagnosed malaria more than those who had other occupations and this could be due to the fact that most of the civil servants claimed to have known more about malaria through mass media which they said is easily accessible to them.

In Table 2 we found out that the level of education attained by the studied participants greatly influences their ability to self-diagnosed malaria. They was statistical significance difference between educational status and self-diagnosing malaria as $p<0.05$. Out of the 69 participants who gave a successfully self-diagnoses, those who had attained primary level of education shows the lists diagnosis compare to those who had attained secondary level. This can be due to the fact that those with secondary level might have acquired much knowledge in school concerning the disease. Also, those who had attained university level had a high frequency of diagnosis compare to those who had attained primary level and this could still be linked to their knowledge acquired from school about the disease. The fact that those who had attained secondary level of education had high frequency in self-diagnosing malaria compare to those who had attained university level could be due to the fact that they represented the highest frequency (proportion) of the studied participants.

In Table 3 where we see the ability of participants to self-diagnose malaria based on the different age groups of participants. They were no statistical significance difference between age and self-diagnosing malaria. This means that participants had equal chances of selfdiagnosing malaria irrespective of their different ages. This could be due to the fact that this study took place in the Regional capital of the South West Region and through mass media people may have had basic information about the disease including it diagnosis.

In Table 4, we see the ability of participants to self-diagnosed malaria based on gender. Also, participants had equal chances to self-diagnose malaria based on sex. That is, they were no statistical significance difference between gender and self-diagnosing malaria. This could be attributed to the recent distribution of mosquito nets in 2011 which may have prompted people to inquire more about the disease and it diagnosis.

This study also revealed that, all the respondents knew at least a clinical sign and symptoms of the disease.

Table 6 and 5 shows the frequency of the signs and symptoms of the disease as mentioned by the studied participants, with the most recognized signs and symptoms been raised body temperature with a percentage of $31.4 \%$. A percentage of $23.8 \%$ participants gave other signs 
South American Journal of Public Health

Special Edition May 2016

and symptoms which were not on the questionnaire, $21.9 \%$ mentioned headache, $11.4 \%$ mentioned body pains and $11.4 \%$ mentioned body seating. These are similar to the signs and symptoms which were reported by the Liberian children and mothers, as well as those reported by Adangbe people in Rural Ghana. Unlike the report of Dickson and others carried out in the North West Region of Cameroon where $2.4 \%$ of the participants did not know any signs and symptoms of the disease, all the respondents in this study knew at least a sign and symptoms of the disease and this could be due to the following reasons: Limbe is the Regional headquarter of the South West Region with a high level of literacy. The high prevalence of malaria in Cameroon, may also be a contributing factor to the general high knowledge about the disease in this area, the smaller sample size of 105 used in this study with respect to 253 participants used by Dickson and others may also be a contributing factor to the increase knowledge of participants about the disease in this study. Also the recent distribution of mosquito nets in the country and the influence of mass media may also be a contributing factor to the high knowledge of the studied participants on the clinical signs and symptoms of the disease.

Of the 105 studied participants who actually took part in this study, 75 of them knew the true cause of the disease to be Plasmodium parasite. This gives a percentage of $71.4 \%$. Other causes as stated by the participants were insects with a percentage of $20 \%$, flies $4.8 \%$ and $3.8 \%$ of the studied participants gave other causes which were not written on the structured questioned. They was a statistical significance difference between various studied participants level of education attained and knowledge the true cause of the disease as shown in Table 5. The discrepancy between educational status and knowing the true caused of malaria with secondary level recording the highest frequency compare to primary level in this study may be due to the knowledge they had acquired in school about the disease. The high knowledge of secondary level compare to university level can be due to the fact that they had the highest proportion of the studied participants. The high knowledge of the studied participant on the cause of the disease differ with the misconceptions about the cause of malaria in Hoima district in Uganda as community members' reported causes to be; Bedbugs, poor nutrition, maize and mangoes. This could be due to increase literacy level among my studied participants. This percentage $(71.4 \%)$ of people knowledge on the cause of malaria also differs with the $90 \%$ knowledge of care givers in Kampala city in Uganda about the cause of the disease. This could equally be due to the fact that, Cameroon is one of the endemic nations of high malaria prevalence, accounting for $40-45 \%$ hospital consultation, $57 \%$ hospitalization and 33\% mortality, which means that malaria is a constant public health problem in this area. Such area may regard the disease as part of their normal life, and thus not consider it to be a health problem. In this study, they were a higher percentage (71.4\%) of participants who knew the true cause of malaria compare to the percentage (27.9\%) recorded in North West Region of the nation. If we assume that the educational status is linked to the profession of the participants, we may suggest that the high knowledge on the cause of malaria was because most respondents who knew the true cause of malaria is being Plasmodium parasite were students. This differs from work of Dickson and others in the North West Region of Cameroon because most of their respondents were labourers. 20\% respondents who reported malaria to be cause by insects and $4.8 \%$ who attributed it to flies may have being due to the fact that they were rains during the period of the study where mosquitoes and flies were reported in some area and so could ascribed respondents to say that they were the cause of it. Also, unhygienic condition in some areas in Limbe which favour breathing of flies and mosquitoes could equally influences respondents to mention insects and flies as the cause of the disease. For the $3.8 \%$ of respondents who gave other causes it suggest that the actual cause of malaria was not well understood.

This study is therefore similar to some works that has been carried out in this field $(17,18)$, on the signs and symptoms that different people use in the diagnosis of the malaria in different areas. It also complement with the work of Dauda in 2004 and Kalisa in 1997 on the knowledge of people about the cause of malaria. It therefore agrees with an increasing 
recognition among health professional that improving the health of poor people depends upon adequate understanding of the socio-cultural aspects of the context in which public health programs are implemented. This study will therefore help people to improve on their ability to diagnose the disease as well as improving their knowledge on the actual cause of the disease as well as it prevention.

\subsubsection{Limitation of the study}

This study has as it limitations the following, which to some extend could contribute to the variation of these findings to previous ones within and out of Cameroon:

- The smaller sample size

- Wrong information probably given by the studied participants

- Manual errors which may have occurred during analysis of the results

\subsection{Conclusion}

This study revealed that $65.7 \%$ of suspected malaria patients who attained Limbe Regional Hospital between the month of May and June successfully self-diagnosed malaria using clinical signs and symptoms. It also revealed that $71.4 \%$ of the studied participants knew the actual cause of malaria to be Plasmodium parasite.

It is not possible to discuss with certainty, the factors that can improve people's ability to self-diagnose malaria, factors that may help people to know clinical signs and symptoms of the disease, as well as knowing the actual cause of it. Educational status of people, the prevalence of the disease in the entire nation and the occupations of people seem to have played major roles.

\subsection{Recommendations}

Given the fact that malaria is still a major health problem in the nation Cameroon $(7,8)$ it will be important that;

- Competent authorities should intensify efforts and implement proper health education messages on malaria determinants such as symptoms in order to improve upon the understanding of malaria in the nation.

- It is suggested that medical practisionals within and out of the nation should take the diagnosis of malaria as a major challenge.

- Also, it will be important for this study to be carried out in other areas within the nation.

\section{References}

[1.] Amino R, Thiberge S, Martin B et al. quantitative imaging of Plasmodium transmission from mosquito to mammal. Nat Med. Feb (2006); 12(2): 220-224.

[2.] Aikins MK,Pickering H, Greenwood BM (1994). Attitudes to malaria, traditional practices and bed nets (mosquito nets) as vector control measures: a comparative study in five West African Countries. J.Trop. Med Hyg. 97:81-86.

[3.] Agyepong, I.A. (1992) Malaria: Ethnomedical Perceptions and Practice in an Adangbe Farming Community and Implications for Control. Social Science and Medicine, Vol. 35(2): 131-137.

[4.] Ashley M. Vaughan, Ahmed S.I. Aly, Stefan H.I Kappe. Malaria parasite pre-erythrocytic stage infection: Gliding and Hiding Cell Host Microbe. II September (2008); 4(3): 209-218.

[5.] Breman, Joel G. M. S. Alilio, and A. Mills. (2004). "Conquering the Intolerable Burden of malaria: What's New, What's Needed: A Summary American Journal of Tropical Medicine and Hygiene 71 (Suppl 2): 1-15.

[6.] Brain M. Greenwood, David A. Fidock, Denis E. Kyle, Stefan H.I. Kappe, Pedro L. Alonso, Frank H. Collins, Patrick E. Duffy. Malaria: progress, perils and prospects for eradication J. Clin. Invest. (2008) 118:1266-1276. Doi: 10.1172/JC133996.

[7.] Centers for Diseases control (CDC). (2006). "Schema of the life cycle of malaria. Accessed 2007 Feb 9. 
South American Journal of Public Health

Special Edition May 2016

[8.] Cecil textbook of medicine, XIX edn (1992). Philadelphia, USA: W.B. Saunders Co,pp. 16901692.

[9.] Carolina Barillas-Mury, Sanjeev Kumar. Plasmodium mosquito intereactions: a tale of dangerous liaisons. Cellular Microbiology (2005); 7(11):1539-1445.

[10.] Dauda Waiswa Batega. (2004). Knowledge attitudes and practices about malaria treatment and prevention in Uganda. Health Communication Partnership based at Johns Hopkins Bloomberg School of. Public Health/Centre for Communication Programs.

[11.] Dickson Shey Nsagha I, Anna Longdoh Njunda, Henri Lucien Fouamno Kamga, Jules Clement Bguedia Assob, Charles Shey Wiysonge, Sarah Mboshi Nsagha, and Alfred Kongnyu Njamnshi (2011). Knowledge and practices relating to malaria: Signs and symptoms, causes and prevention; Journal of Public Health and Epidemiology Vol. 3(6).pp. 294-300, June 2011.

[12.] Eric A. Achidi, Tobias O Apinjoh, Judith K Anchang-Kimbi, Regina N Mugri, Andre N Ngwai, and Clarisse N Yafi (2012). Severe and uncomplicated falciparum malaria in children from three regions and three ethnic groups in Cameroon: prospective study. Achidi et al. malaria Journal 2012. $11: 215$.

[13.] Einterz EM (2003). Perceptions of malaria transmission, presentation and management in northern Cameroon. Trans. R. Soc. Trop. Med. Hyg. 97: 51-51.

[14.] Edmondson Jane. (2001) page 24. Malaria and Poverty: Opportunities to Address Malaria through Debt Relief and Poverty Reduction Strategies; Working document prepare by Malaria Consortium.Pg.24.

[15.] Foster S(1995). Treatment of malaria outside the formal health services. J Trop.Med.Hyg. 98:2934.

[16.] Greenberg AE, Ntumbanzondo CM, Malaria L, Howell J, Davachi F (1989). Hospital-based Surveillance of malaria-related Pediatric morbidity and mortality in Kinshasa, Zaire. WHO Bull. 67:89196.

[17.] Global Malaria Action Plan (2011). Available at http://www.rollbackmalaria.or/gmap and $\mathrm{http}: / /$ www.rollbackmalaria.org/gmap/3-2html.

Source: Malaria Prevalence Model: I. Kleinschmidt (2001). An empirical malaria distribution map for West Africa. Tropical Medicine and International Health 6:779-786. Topographical data.

[18.] Handrickse RG; Hasan AH; Olumide LO and Akinkunmi A. (1971). Malaria in early childhood. Annals of Tropical Medicine and Parasitology (1971); 65:1-20.

[19.] Heather M Ferguson, Andrew F Read, Mosquito appetite for blood is stimulated by Plasmodium chabaudi infections in themselves and their vertebrate hosts. Malaria Journal (2004); 3:12doi: 10.1186/1475-2875-3.

[20.] Isaac Keango Nyamongo. (1998) lay people's responses to illness: An Ethnographic study of antimalaria behavior among the Abagusii of Southwestern Kenya.

http:/www.who.int/malaria/world_malaria_report_(2010)/en/index.html.

[21.] Ikome LE, Ndamukong KJ, Kimbi H (2002) Prevalence and case-control study of cerebral malaria in Limbe of the South West Cameroon. Afr J Health Sci 9:61-67.

[22.] Jackson, L.C. (1985) malaria in Liberian children and mothers: Biocultural Perceptions of illness Vs. clinical Evidence of Disease. Social Science and Medicine, Vol 20(12): 1281-1287.

[23.] Jake Baum, Dave Richard, Julie Heale et al. a conserved Molecular Motor Drives Cell Invasion and Gliding Motility across Malaria Life Cycle Stages and Other Apicomplexan Parasites. The Journal of Biological Chemistry. Febraury (2006); 281:5197-5208.

[24.] Kalisa, M. W. (1997). A study of malaria control policy in Hoima district (Unpublished). Ministry of Gender and Community Development, Kampala, Uganda. J. Trop Med. Hyg. 97:81-86.

[25.] Kun JF, Mission MA,Lell B, Sovric M, Knoop H, et al. (2002) New emerging Plasmodium falciparum genotypes in children during the transition phase from asymptomatic parasitemia to malaria. AM J Trop Med HYG 66:653-658.

[26.] Kimbi HK, Awah NW, Ndamukong KJ, Mbuh JV (2005) Malaria infection and its consequences in school children. East Afr Med J 83:92-97.

[27.] Kebaier C, Voza T, Vanderberg J. Kinetics of Mosquito-Injected Plasmodium Sporozoites in Mice: Fewer Sporozoites Are Injected into Sporozoite-Immunised Mice. Plos Pathog (2009); 5(4): e1000399. 
[28.] Langhorne J, Ndungu FM, Sponaas A, Marsh K: Immunity to malaria: more questions than answers, Nat Immunol (2008), 9:725-732.

[29.] Laurence Floren, Michael P. Washburn, J. Dale Raine et al. a proteomic view of the Plasmodium falciparum life cycle Nature October (2002); 419:520-526.

[30.] Lucy Megumi Yamauchi, Alida Coppi, Georges Snounou, Photini Sinnis. Plasmodium sporozoites trickle out of the injection site. Cell Microbiol. 1 May (2007); 9(5):1215 - 1222.

[31.] Malanet P. Speilman A, Sachs J: (2004). The malaria gap. Am J Trop Med Hyg (2004), 71:141 146

[32.] Ministère de la Santé Publique (2007). Plan stratégie nationale de la lute contre le paludisme au Cameoun. Pp 1- 134.

[33.] Michael F Good, Denise L Doolan. Malaria's journal through the lymph node. Nature Medicine (2007); 13:1023-1024

[34.] Miguel Prudêncio, Ana Rodriguez, Maria M. Mota. The silent path to thousands of merozoites: the Plasmodium liver stage. Nature Reviews Microbiology (2006); 4:849-856.

[35.] Njama D, Dorsey G, et'al (2003). Urban malaria: primary caregivers' knowledge, attitudes, practices and predicators of malaria incidence in a cohort of Uganda children. In Trop Med and Int Health. Vol 8 of No 8 pp: 685-692.

[36.] Olivier Silvie, Maria M Nota, Kai Matuschewski, Miguel Prudencio. Interactions of the malaria parasite and its mammalian host. Current Opinion in Microbiology (2008); 11:352-359.

[37.] Quakyi AI, Leke RG, Befidi-Mengue R, Tsafack M, Bomba-Nkolo D, Manga, L, Tchinda V, Njeugue E, Kouontchou S, Fogako J, Nyonglema P, Harun LT, Djokam R, Sama G, Eno A, Megnekou R, Metenou S, Ndoutse L, Same-Ekobe A, Alake G, Meli J, Ngu J, Tietche F, Lohoue J, Mvondo, J.L, Wansi E, Leke R, Folefack A, Bigoga J, Bomba-Nkolo C, Titanji V, Walker-Abbey A, Hickey AM, Johnson AH, and Taylor, DW. (2000); The Epidemiology of Plasmoium falciparum in two Cameroonian villages: Simbok and Etoa. American Journal of Tropical Medical and Hygiene. (2000); 5:222-230.

[38.] Quakyi AI; Leke RG; Befidi-Mengue R; Tsafack M; Bomba-Nkolo D; Manga, L; Tchinda V; Njeugue E; Kountchou S; Fogako J; Nyonglema P; Harun LT; Djokam R; Sama G; Eno A; Megnekou R; Metenou S; Ndoutse L; Same-Ekobe A; Alake G; Meli J; Ngu J; Tietche F; Lohoue J; Mvondo, JL; Wansi E; Leke R; Folefack A; Bigoga J; Bomba-Nkolo C; Titanji V; Walker-Abbey A; Hickey AM; Johnson AH; and Taylor DW. The epidemiology of plasmodium falciparum in two Cameroonian villages: Simbok and Etoa. American Journal of Tropical Medicine and Hygiene (2000); 5:222-230.

[39.] Roll Back Malaria Partnership. (2002). Malaria and children. World Heal Organization Accessed May 2, 2010.

[40.] Snow RW, Guerra CA, Noor AM, Myint HY, Hay SI: The global distribution of clinical episodes of plasmodium faciparum malaria. Nature (2005), 434:214 - 217.

[41.] Smith DC (1982). The rise and fall of typhomalaria fever. I: origin. J. Hist. Med. Allied Sci, 37:182-220.

[42.] Snow RW, Guerra CA, Abdisalan M; Myint HY, Hay SI, (2005): The global distribution of clinical episodes of Plasmodium falciparum malaria. Nature 2005, 343:214-217.

[43.] Samba E; (2001). The malaria burden and Africa. AM J Trop Med Hyg, 64 (1 - 2 Suppl): ii.Preface.

[44.] Sylvia Münter, Benedikt Sabass, Christine Selhuber-Unke et al. plasmodium sporozoite Motility is Modulated by the Turnover of Discrete Adhesion Sites Cell Host and Microbe. December (2009); 6(17):551-562.

[45.] Theresia Nkuo-Akenji, Nelson N. Ntonifor, Maize B. Ndukum. Helen K. Kimbi, Edith L. Abongwa, Armand Nkwescheu, Damain N. Anong, Michael Songmbe, Michael G. Boyo, Keneath N, Ndamukong and Vincent P.K Titanji. (2006). Environmental factors affecting malaria parasite prevalence in rural Bolifamba, South West Cameroon. TN environment (2004). 71:141 146.

[46.] United Nations Statistics Division (2011). Malaria mortality in Cameroon. Available at http:/www.indexmundi.com/Cameroon/malaria-mortality.html. Retrieved, ay 11.

[47.] World Health Organization, World Malaria report, Global malaria programme (2011). Geneva World Health Organization Publication; (2011) 
South American Journal of Public Health

Special Edition May 2016

[48.] World Economic Forum (2002). Global health initiative. Private sector intervention case example Chad/Cameroon oil pipeline. Using malaria control strategically to improve worker's health and prevent project delay,pp 108.

[49.] WHO:World Malaria report, (2011). Global malaria programme. Geneva World Health Organization Publication; (2011)

[50.] World Health Organization. (2002). World Health Report Reducing Risks, Promoting Health Life.Pg 77. Geneva.

[51.] WHO World Malaria Report (2010): Available at http//whqlibdoc.who.int/publications/2010/9789241564106_eng.pdf 